\title{
Análisis biomecánico de dos constructos de osteosíntesis en fracturas oblicuas largas de primer metatarsiano
}

\author{
Biomechanical analysis of two osteosynthesis constructs \\ in long oblique fractures of first metatarsal
}

\author{
Francisco Guillermo Castillo-Vázquez, ${ }^{\star}$ Juan Carlos Hermida Ochoa, ${ }^{\ddagger}$ Luis Felipe Hermida Galindo§
}

Citar como: Castillo-Vázquez FG, Hermida OJC, Hermida GLF. Análisis biomecánico de dos constructos de osteosíntesis en fracturas oblicuas largas de primer metatarsiano. An Med ABC. 2021; 66 (2): 97-103. https://dx.doi.org/10.35366/100477

\section{RESUMEN}

Introducción: El primer metatarsiano es una estructura anatómica fundamental para la bipedestación y la marcha humana, por lo que las fracturas desplazadas del mismo pueden dar lugar a dolor, úlceras plantares, limitación funcional y disminución en la calidad de vida del individuo. Es por esto que conocer la fijación ideal de la misma puede asegurar un mejor pronóstico para los pacientes con esta lesión. Objetivo: Debido a que la literatura equipara el uso de tornillos de compresión con el uso de un tornillo de compresión más placa de protección en su tratamiento y a que no se encontró un estudio biomecánico que soporte esta afirmación, decidimos realizar pruebas biomecánicas que permitan corroborar o refutar esa teoría. Material y métodos: Se utilizaron metatarsianos de cerdo en los que se simuló una fractura en laboratorio y que fueron fijados con las dos técnicas de osteosíntesis previamente mencionadas para definir la equiparabilidad biomecánica de las mismas. Se realizaron las pruebas biomecánicas en los dos constructos previamente descritos sometiéndose a una fuerza de $250 \mathrm{~N}$ por 300,000 ciclos. Resultados: Nuestro estudio demuestra la superioridad del constructo de dos tornillos mediante pruebas biomecánicas, así como una menor pérdida de la rigidez durante los ciclos de carga estudiados. Conclusiones: Los tornillos de compresión son biomecánicamente superiores a un tornillo más una placa de protección en fracturas oblicuas largas de primer metatarsiano.

\section{ABSTRACT}

Introduction: The first metatarsal is a fundamental anatomical structure for bipedestation and walking so displaced fractures can lead to pain, plantar ulcers, functional limitation, and decreased quality of life of the individual. Therefore knowing the ideal fixation of it can ensure a better prognosis for patients with this lesion. Objective: Because the literature equates the use of compression screws with the use of a compression screw plus protection plate in its treatment and because no biomechanical study was found to support this claim, we decided to perform biomechanical tests to corroborate or refute that theory. Material and methods: Pork metatarsals were used in which a laboratory fracture was simulated, and which were fixed with the two osteosynthesis techniques mentioned above to define the biomechanical equality of them. Biomechanical testing was performed on the two constructs described above undergoing a force of $250 \mathrm{~N}$ per 300,000 cycles. Results: Our study demonstrates the superiority of the construct of two screws by biomechanical tests a less loss of rigidity of the same during the load cycles. Conclusions: Compression screws are biomechanically superior to a screw plus a protection plate in first metatarsal long oblique fractures.
* Médico Residente de Ortopedia del Centro Médico ABC. México.

₹ Cirujano de Columna, Jefe del Centro de Investigación y Laboratorio de Biomecánica (CILAB), Hospital de Ortopedia para Niños «Dr. Germán Díaz Lombardo". México.

$\S$ Cirujano de Pie y Tobillo, Médico Staff del Centro Médico ABC. México.
Recibido: 01/04/2021. Aceptado: 03/06/2021.

Correspondencia:

Dr. Francisco Guillermo Castillo-Vázquez

E-mail: castillo.ortopedia@gmail.com 
Palabras clave: Metatarsiano, prueba biomecánica, osteosíntesis.

Nivel de evidencia: III

\section{INTRODUCCIÓN}

El pie es una estructura altamente especializada que le permite al ser humano mantener una posición erecta, con lo que es capaz de hacer uso de sus extremidades superiores para funciones desarrolladas, además de propulsarlo para poder desplazarse, este depende de una perfecta integridad estructural entre sus partes para llevar a cabo sus funciones de forma adecuada. ${ }^{1,2}$ De manera particular, el primer metatarsiano está apoyado sobre los dos metatarsianos que en conjunto dan una superficie de carga de 2.5 $\mathrm{cm}^{2}$, sobre la cual descansa el doble de peso que sobre el resto de los metatarsianos durante la fase de apoyo (aproximadamente 30\% del peso corporal en el punto de carga máxima), por lo que cualquier alteración en su estructura y en su función tendrá un grave impacto sobre la biomecánica del pie en su totalidad..$^{3-6}$ Las fracturas son una patología que de forma súbita puede alterar la organización estructural del primer metatarsiano; aquéllas que ocurren en metatarsianos forman una tercera parte de todas las fracturas del pie y el 5\% de todas las fracturas del esqueleto. ${ }^{5,7}$

La mayoría de los textos concuerdan en definir los criterios quirúrgicos de las fracturas diafisarias del primer metatarsiano como: fracturas expuestas, conminutas, extensión articular, angulaciones diafisarias mayores a 10 grados en el plano sagital y/o aquéllas con un acortamiento mayor o igual a $4 \mathrm{mi}$ límetros. Además, estos dos últimos parámetros son utilizados para clasificar dichas fracturas como inestables debido al alto riesgo de desplazamiento generado por los músculos intrínsecos y extrínsecos del Hallux en relación con el hueso. ${ }^{2-9}$

En cuanto al tratamiento quirúrgico, existe un gran número de constructos de osteosíntesis propuestos para el tratamiento de este tipo de fracturas, de los cuales son aceptados el uso de clavillos cruzados e intramedulares, tornillos de compresión, un tornillo de compresión asociado a una placa de protección y placas bloqueadas de bajo perfil, considerando todos, salvo el caso de los clavillos, como equivalentes. ${ }^{2-10}$

Se realizó una búsqueda en la literatura considerando como criterios de inclusión el ser artículos que utilizarán pruebas biomecánicas con sometimiento
Keywords: Metatarsal, biomechanical test, osteosynthesis.

Level of evidence: III

de los constructos a fuerzas simulando la marcha, que utilizaran implantes de osteosíntesis y que fueran realizadas en modelos de primer metatarsiano, sin encontrarse estudios biomecánicos que comparen estos implantes en dicha región del organismo con las cargas específicas a las que está sometido el primer metatarsiano y que permitan definir si alguno es superior a los demás; ${ }^{11-23}$ es por esto que decidimos realizar cargas cíclicas, simulando la marcha humana en metatarsianos con fractura en laboratorio y que fueron fijados con las dos técnicas de osteosíntesis previamente mencionadas para definir la equiparabilidad biomecánica de las mismas.

Objetivo: la intención de este estudio es conocer, mediante pruebas mecánicas, si alguno de los constructos biomecánicos comparados ofrece mayor rigidez y estabilidad que otro.

\section{MATERIAL Y MÉTODOS}

Para este estudio fue utilizado el simulador de movimiento articular de seis grados de libertad AMTI $\mathrm{VIVO}^{\mathrm{TM}}$, que asemeja la cinética y cinemática de los movimientos corporales a las condiciones en vivo. Es la única maquinaria en el mundo que permite 6 grados de libertad y recrear movimientos complejos para probar diferentes características de especímenes biológicos. Además, es el primer simulador en ser aceptado por las mayores organizaciones biomecánicas como un estándar de pruebas.

Todas las disecciones, constructos de osteosíntesis y pruebas biomecánicas se realizaron en el Centro de Investigación y Laboratorio de Biomecánica del Hospital «Dr. Germán Díaz Lombardo». Se obtuvieron piezas biológicas de cerdo de la raza large white, las cuales se conservaron a $-25{ }^{\circ} \mathrm{C}$ durante el resto del tiempo previo a la disección. ${ }^{24-28}$ Se realizó la disección de éstas, obteniéndose de forma íntegra el segundo y tercer metatarsianos de manera bilateral; se procedió a realizar una osteotomía en las diáfisis de las cuatro piezas, simulando una fractura oblicua larga con mediciones consistentes para evitar la variación del trazo entre piezas (osteotomía a 30 grados del eje longitudinal del metatarsiano de dorsal-proximal a plantar-distal con una longitud de 36 milíme- 

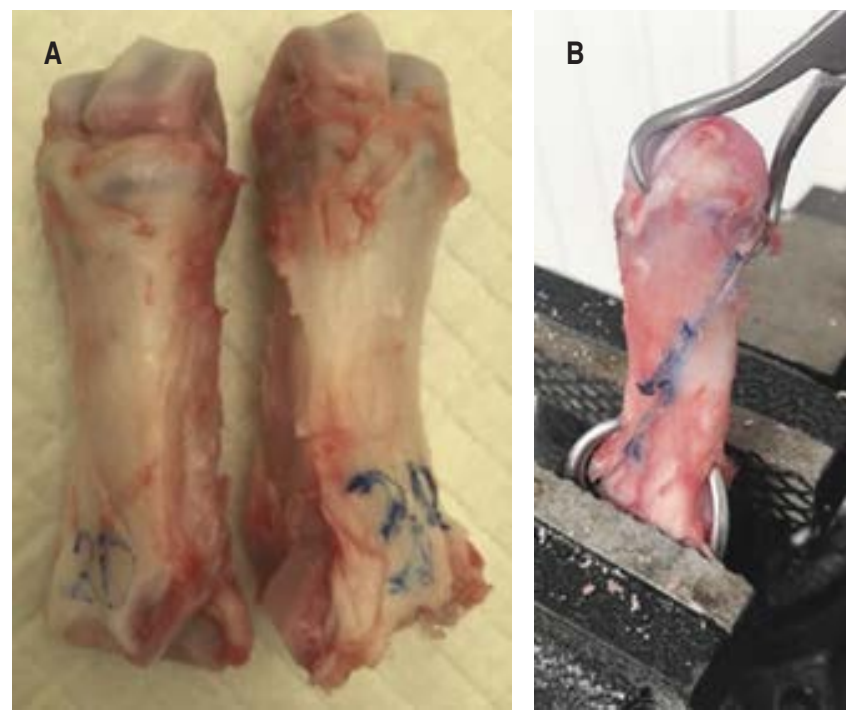

Figura 1: A) Segundo y tercer metatarsianos de cerdo disecados e identificados. B) Montaje del metatarsiano en el que se aprecia el corte de 36 milímetros realizado en la diáfisis del metatarsiano de dorsal a plantar y de proximal a distal a 30 grados del eje longitudinal del mismo.

tros) (Figura 1). Después de las osteotomías, se realizó la fijación con dos constructos de osteosíntesis:

1. Tornillos de compresión: se colocaron dos tornillos corticales de 3.5 milímetros perpendiculares al trazo de fractura en la unión de los tercios proximal-medio y medio-distal de la osteotomía con técnica de canal liso hasta lograr una adecuada compresión del trazo, rotando a fuerza máxima con dos dedos de la mano no dominante y apreciándose un cierre adecuado del trazo de fractura (Figura 2A).

2. Tornillo de compresión + placa de protección: se colocó un tornillo cortical de 3.5 milímetros mediante técnica de canal liso perpendicular al trazo de fractura en el control de éste en el plano sagital posterior. Se protegió al constructo con una placa tercio de caña de cinco orificios colocada en cortical medial fijada con dos tornillos proximales $\mathrm{y}$ dos distales al trazo de fractura (Figura $2 B$ ).

$\mathrm{Al}$ encontrar los valores correctos de momento y desplazamiento necesarios para ejercer la fuerza requerida, así como la posición ideal de colocación, se procedió a fijar cada constructo de la misma forma, después fueron sometidos a la carga previamente determinada de forma cíclica hasta la fatiga o hasta los 300,000 ciclos, que son las cargas a las que está sometido el metatarsiano durante seis semanas, es decir, hasta la consolidación del trazo de fractura (Figura 3). 3,4,13-23,29-42

\section{RESULTADOS}

Se realizaron las pruebas biomecánicas en los tres constructos previamente descritos, sometiéndolos a una fuerza de $250 \mathrm{~N}$ durante 300,000 ciclos y se realizó un registro de la pérdida de fuerza y el torque resultante cada 100 ciclos.

Tornillos de compresión: se obtuvieron los datos horarios a partir de los 11 minutos de prueba y, de acuerdo con los parámetros obtenidos, se procedió a graficar los resultados en una tabla de pérdida de fuerza (Figura 4) en la que se apreció un deterioro en la resistencia del constructo al desplazamiento utilizado de $56.28 \mathrm{~N}$, lo cual correspondió al $26.13 \%$ de la resistencia inicial; vale la pena mencionar que $6 \%$ de esta fuerza (Figura 5) se perdió durante la primera hora de la prueba, durante las siguientes 12 horas se apreció una pérdida de $1 \%$ de la fuerza del constructo por hora, seguido por un periodo de meseta de 13 horas durante el cual sólo se perdió 5\% de la
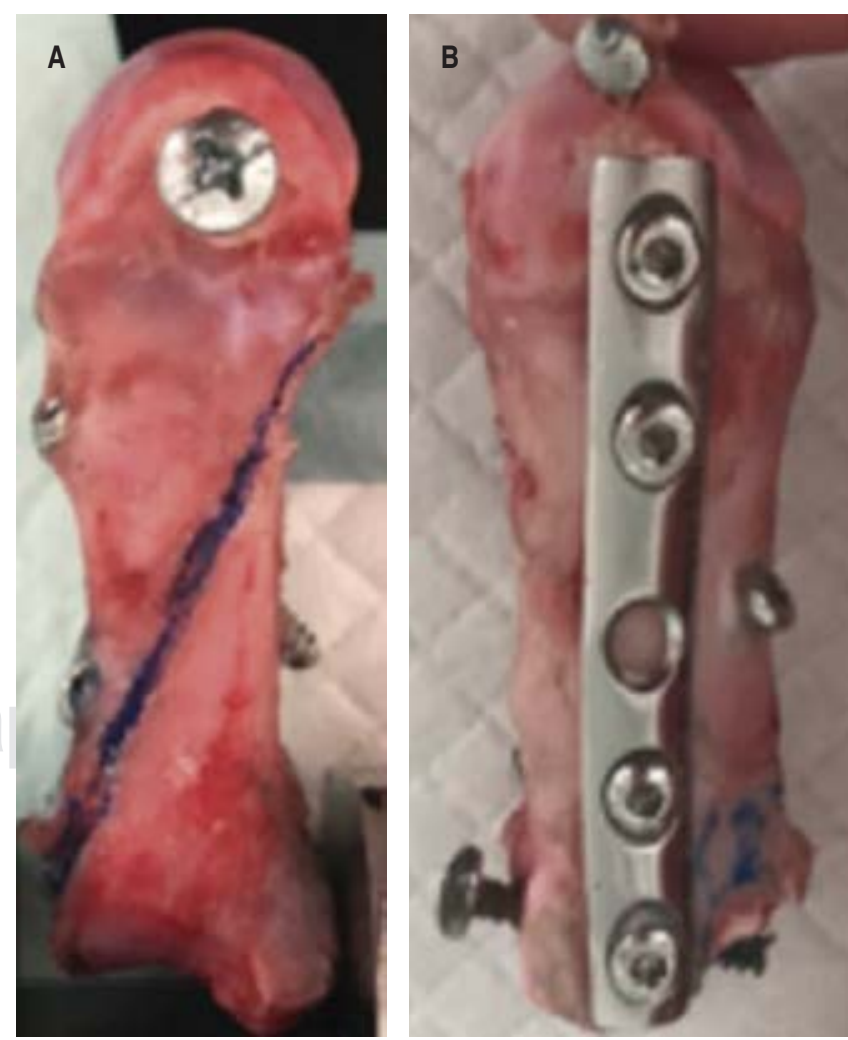

Figura 2: A) Tornillos de compresión. B) Tornillos de compresión + placa tercio de caña de protección. 


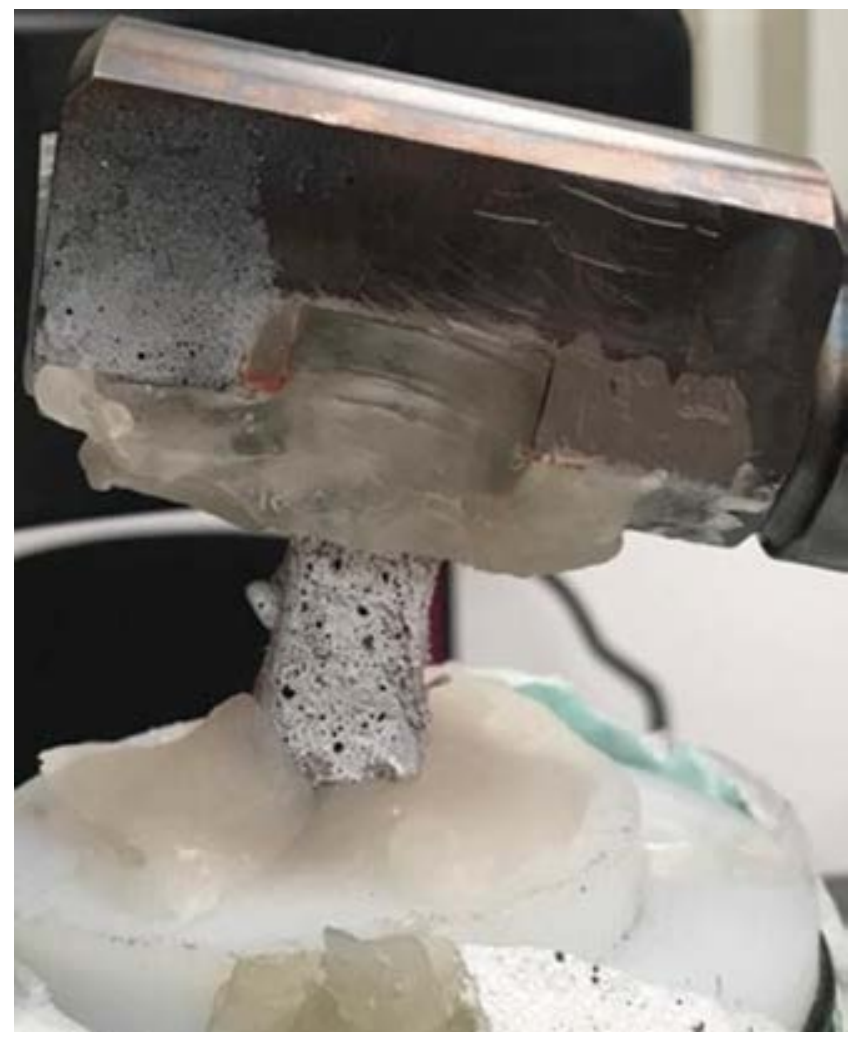

Figura 3: Montaje final del constructo.

resistencia, después se apreció una recuperación momentánea de $1 \%$ de la fuerza durante cinco horas, lo que podemos traducir como el efecto de pandeo o buckling, ${ }^{43}$ que se refiere a la cohesión molecular generada previa al momento de la falla de un constructo debido a la disminución de la distancia entre sus moléculas, lo que genera un aumento temporal de la rigidez del constructo a la fuerza aplicada que cederá con el paso del tiempo para dar lugar a la falla del mismo. Por último, se registró una nueva meseta durante las 11 horas restantes de la prueba, en la que se perdió 3\% de la resistencia total sin llegar al punto de fatiga del constructo.

Tornillos de compresión más placa de protección: se realizó el mismo análisis de datos que para los tornillos de compresión, de tal forma que pudieran ser comparados. De manera similar al constructo de dos tornillos, durante la primera hora hubo una pérdida súbita de la resistencia de $25 \mathrm{~N}$, lo cual fue equivalente a $11 \%$ de la pérdida de resistencia del constructo o $114.06 \mathrm{~N}$ (Figura 6), y a 25\% de la pérdida de fuerza total; esta caída continuó siendo considerable durante la segunda hora, en la que se perdió 5\% de la resistencia y $3 \%$ en la tercera hora, seguido por un periodo de meseta de seis horas, tiempo en el que sólo se perdió $2 \%$ de la fuerza, continuando con un descenso de $1 \%$ por hora durante las siguientes nueve horas y una nueva meseta de 17 horas sin pérdida de fuerza, finalizando con un periodo de siete horas en las que se perdió menos de $1 \%$ de la resistencia por hora. No se logró llegar a la fatiga del material; sin embargo, en comparación con el constructo de dos tornillos de compresión, la pérdida de fuerza fue notoria en mayor medida con un $20 \%$ más de pérdida de resistencia en el mismo periodo de tiempo y con la misma carga, mostrando además una pendiente más pronunciada y continua que el primer constructo descrito (Figura 7).

\section{DISCUSIÓN}

Una de las metas del estudio presentado fue valorar si dos tornillos de compresión se desempeñaban igual de bien que un tornillo de protección, asociado a una

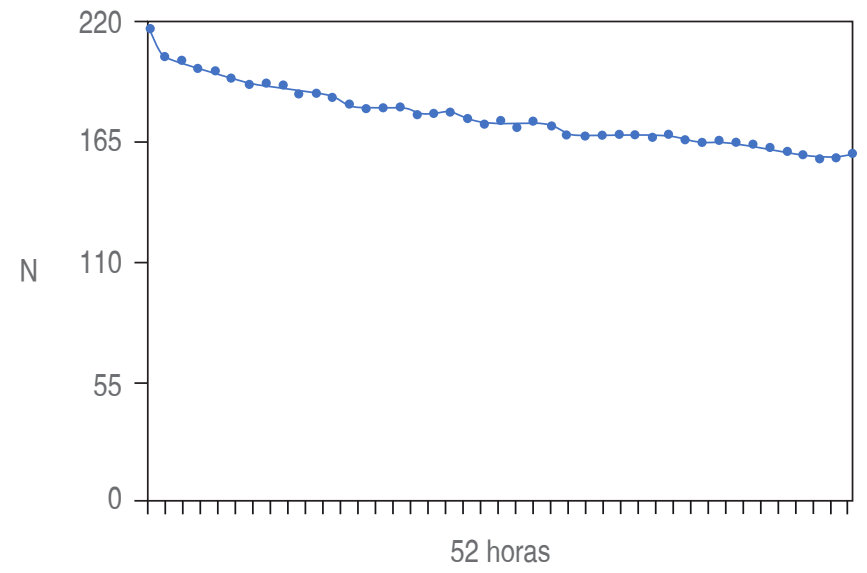

Figura 4: Pérdida de la fuerza por hora (7,000 ciclos) de dos tornillos de compresión en un trazo oblicuo largo de primer metatarsiano.

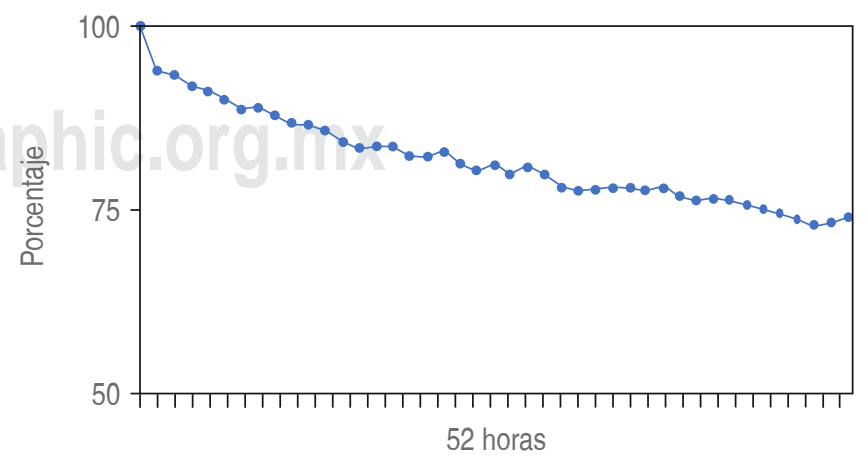

Figura 5: Porcentaje de pérdida de la fuerza por hora de dos tornillos de compresión en un trazo oblicuo largo de primer metatarsiano. 


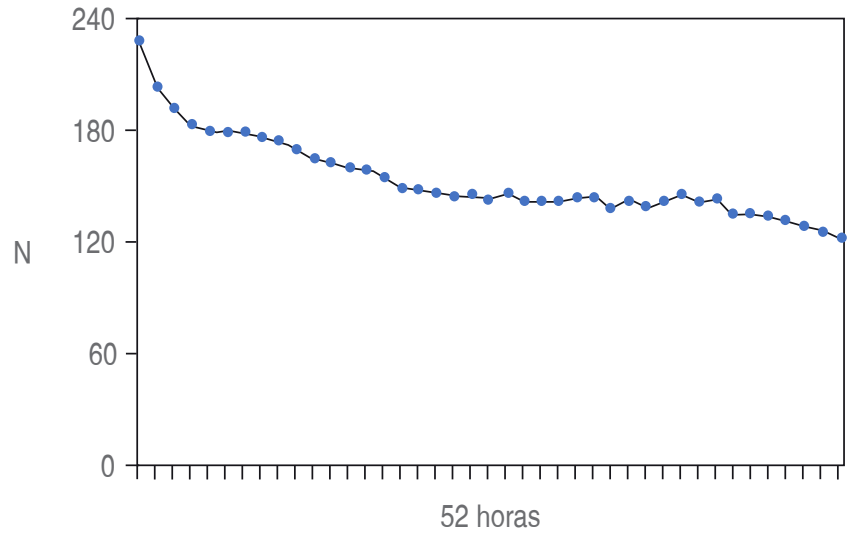

Figura 6: Pérdida de la fuerza por hora de un tornillo de compresión más una placa de protección en un trazo oblicuo largo de primer metatarsiano.

placa de protección en la fijación de fracturas oblicuas largas de primer metatarsiano.

Los resultados de nuestra investigación demuestran que no sólo son equiparables, sino que los tornillos de compresión superan biomecánicamente al segundo constructo en cuanto a rigidez, determinada tanto por la fuerza que se requiere para poder desplazarlo, como por la deformación que sufre el trazo durante la carga, lo cual podría traducirse como una menor posibilidad de desplazamiento, que es una de las principales complicaciones de este tipo de fracturas y una variable que se pretende resolver con el tratamiento quirúrgico.

Con esta información en mente, podemos determinar que la colocación de un tornillo de compresión a través de nuestro trazo de fractura sería insuficiente para evitar el desplazamiento de nuestro trazo sometido a cargas fisiológicas, por lo que nuestra discusión en este punto se reduciría a definir qué implante neutralizaría de mejor manera las fuerzas deformantes sobre nuestro tornillo. Mientras que un segundo tornillo de compresión, a través del trazo de fractura, parecería inferior a una placa de protección, aunque presenta ventajas en varios aspectos; el colocar un segundo tornillo de compresión dentro del trazo de fractura y perpendicular a las fuerzas deformantes lo sitúa como un implante con una ventaja posicional sobre una placa colocada en la superficie medial del hueso que, por ende, no se encuentra en el plano en el que se requieren neutralizar las fuerzas, por lo tanto, a pesar de contar con más densidad de tornillos, se encuentran en desventaja al aplicar fuerzas en el plano sagital del hueso, lo cual ya ha sido descrito en la literatura. ${ }^{44}$

Podemos definir la superioridad del constructo de dos tornillos en dos aspectos; Betts ${ }^{45}$ menciona, como parte de su revisión sobre regulaciones biomecánicas de la consolidación ósea, que la alteración en la vascularidad ósea es el principal factor determinante por sobre la estabilidad mecánica, retomando el menor abordaje y desperiostización necesaria para la colocación de dos tornillos interfragmentarios que permiten una mayor vascularidad de los fragmentos óseos. Por otro lado, nuestro análisis nos permitió observar durante cada carga cíclica cómo la colocación medial de la placa la convertía en un fulcro que evitaba el movimiento medial del trazo, pero exageraba su movimiento en la cortical lateral, situación que queda en evidencia al apreciar un desplazamiento mayor al doble que el observado en el constructo de los dos tornillos, por lo que la placa no sólo es inferior, sino que no se recomienda para evitar el movimiento del trazo, ya que su posición en el hueso la convierte en un implante que favorece el movimiento interfragmentario en el trazo de fractura en la cortical opuesta, inestabilizando aún más el constructo.

\section{CONCLUSIÓN}

Las fracturas de primer metatarsiano, a pesar de no ser frecuentes, representan un problema clínico grave para aquéllos que la padecen por su potencial para presentar importantes complicaciones en la transferencia de cargas, la biomecánica de la marcha y los tejidos blandos adyacentes, por lo que es crucial conocer el mejor tratamiento para este tipo de fracturas. Dentro del tratamiento quirúrgico, la literatura menciona los tornillos de compresión y el uso de un tornillo de compresión asociado con una placa de protección como biomecánicamente equivalentes.

Este estudio demuestra la superioridad del constructo de dos tornillos mediante pruebas biomecá-

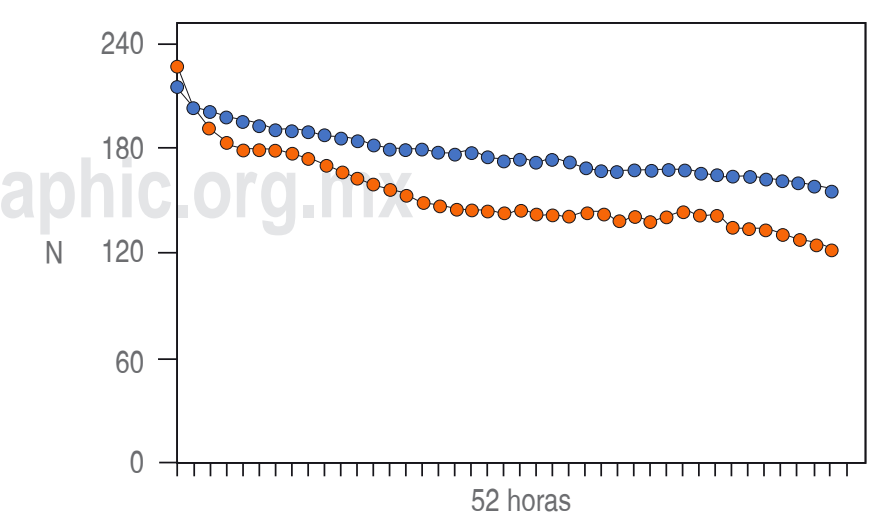

Figura 7: Comparativa de pérdida de fuerza por hora entre dos tornillos de compresión (azul) y tornillo de compresión más placa de protección. 
nicas y análisis interferométrico, observándose una menor pérdida de la rigidez durante los ciclos de carga necesarios para la fatiga, así como una menor deformación del trazo de fractura durante la carga, asociado con la necesidad de un menor abordaje, una menor desperiostización y un menor costo para el paciente, colocándolo como el mejor constructo de osteosíntesis en trazos oblicuos largos de primer metatarsiano. Es importante mencionar que, a pesar de su ventaja biomecánica, no es capaz de mantener una estabilidad suficiente para permitir el apoyo completo temprano después de la cirugía, por lo que es mandatorio proteger el apoyo de los casos durante las primeras etapas postoperatorias para evitar el riesgo de no unión de nuestro trazo de fractura.

\section{AGRADECIMIENTOS}

A la Dra. Karen Berenice Puello Nakayama por su exhaustiva revisión del manuscrito y sus consejos para la realización del mismo.

\section{REFERENCIAS}

1. Kelikian A, Sarrafian S. Sarrafian's anatomy of the foot and ankle. Descriptive, topographic, functional. 3rd edition. Philadelphia: Wolters Kluwer; 2011.

2. Saltzman C, Anderson R, Coughlin M. Mann's surgery of the foot and ankle. 9th edition. Philadelphia: Elsevier Saunders; 2014.

3. Buckley R, Moran C, Apivatthakakul T, AO Foundation. AO principles of fracture management. 3rd edition. Switzerland. Davos Platz, Switzerland: AO Foundation, Stuttgart, Germany; New York, NY: Distribution by Georg Thieme Verlag; 2017.

4. Rammelt S. Swords M, Dhillon M, Sands A. Manual of fracture management. Foot and ankle. Switzerland: AO Foundation; 2020.

5. Buddecke DE, Polk MA, Barp EA. Metatarsal fractures. Clin Podiatr Med Surg. 2010; 27 (4): 601-624.

6. Boutefnouchet T, Budair B, Backshayesh P, Ali SA. Metatarsal fractures: a review and current concepts. Trauma. 2014; 16 (3): 147-163.

7. Petrisor BA, Ekrol I, Court-Brown C. The epidemiology of metatarsal fractures. Foot Ankle Int. 2006; 27 (3): 172-174.

8. Sarpong NO, Swindell HW, Trupia EP, Vosseller J. Metatarsal fractures. Foot Ankle Orthop. 2018; 3 (3). Available in: https:// doi.org/10.1177/2473011418775094

9. Robinson A, Brodsky J, Negrine J. Core topics in foot and ankle surgery. United Kingdom: Cambridge University Press; 2018.

10. Burr DB, Milgrom C. Musculoskeletal fatigue and stress fractures. Florida: CRC Press; 2001.

11. Chatzistergos PE, Karaoglanis GC, Kourkoulis SK, Tyllianakis M, Stamatis ED. Supplementary medial locking plate fixation of Ludloff osteotomy versus sole lag screw fixation: a biomechanical evaluation. Clin Biomech (Bristol, Avon). 2017; 47: 66-72.

12. Kim JS, Cho HK, Young KW, Kim JS, Lee KT. Biomechanical comparison study of three fixation methods for proximal chevron osteotomy of the first metatarsal in hallux valgus. Clin Orthop Surg. 2017; 9 (4): 514-520.

13. Hofstaetter SG, Glisson RR, Alitz CJ, Trnka HJ, Easley ME. Biomechanical comparison of screws and plates for hallux valgus opening-wedge and Ludloff osteotomies. Clin Biomech (Bristol, Avon). 2008; 23 (1): 101-108.

14. Massengill JB, Alexander H, Langrana N, Mylod A. A phalangeal fracture model--quantitative analysis of rigidity and failure. J Hand Surg Am. 1982; 7 (3): 264-270.

15. Yu X, Pang QJ, Chen XJ. The biomechanical study of the influence to the forefoot plantar pressure of the first tarsometatarsal joint fracture-dislocation fixed by three different implants. Pak J Med Sci. 2017; 33 (1): 146-150.

16. Ochman S, Doht S, Paletta J, Langer M, Raschke MJ, Meffert $\mathrm{RH}$. Comparison between locking and non-locking plates for fixation of metacarpal fractures in an animal model. J Hand Surg Am. 2010; 35 (4): 597-603.

17. Popoff I, Negrine JP, Zecovic M, Svehla M, Walsh WR. The effect of screw type on the biomechanical properties of SCARF and crescentic osteotomies of the first metatarsal. J Foot Ankle Surg. 2003; 42 (3): 161-164.

18. Smith K, Lidtke RH, Oliver NG, Maker JM. Mechanical comparison of cortical screw fixation versus locking plate fixation in first metatarsal base osteotomy. J Foot Ankle Surg. 2014; 53 (5): 529-533.

19. Stamatis ED, Navid DO, Parks BG, Myerson MS. Strength of fixation of Ludloff metatarsal osteotomy utilizing three different types of Kirschner wires: a biomechanical study. Foot Ankle Int. 2003; 24 (10): 805-811.

20. Tsilikas SP, Stamatis ED, Kourkoulis SK, Mitousoudis AS, Chatzistergos PE, Papagelopoulos PJ. Mechanical comparison of two types of fixation for ludloff oblique first metatarsal osteotomy. J Foot Ankle Surg. 2011; 50 (6): 699-702.

21. Unal AM, Baran O, Uzun B, Turan AC. Comparison of screwfixation stabilities of first metatarsal shaft osteotomies: a biomechanical study. Acta Orthop Traumatol Turc. 2010; 44 (1): 70-75

22. Waris E, Ashammakhi N, Raatikainen T, Tormala P, Santavirta $\mathrm{S}$, Konttinen YT. Self-reinforced bioabsorbable versus metallic fixation systems for metacarpal and phalangeal fractures: a biomechanical study. J Hand Surg Am. 2002; 27 (5): 902-909.

23. Trnka HJ, Parks BG, Ivanic G, Chu IT, Easley ME, Schon LC et al. Six first metatarsal shaft osteotomies: mechanical and immobilization comparisons. Clin Orthop Relat Res. 2000; (381): 256-265.

24. Thorup VM, Togersen FA, Jorgensen B, Jensen BR. Biomechanical gait analysis of pigs walking on solid concrete floor. Animal. 2007; 1 (5): 708-715.

25. Decker S, Reifenrath J, Omar M, Krettek C, Muller CW. Nonosteotomy and osteotomy large animal fracture models in orthopedic trauma research. Orthop Rev (Pavia). 2014; 6 (4): 5575.

26. Reichert JC, Saifzadeh S, Wullschleger ME, Epari DR, Schütz MA, Duda GN et al. The challenge of establishing preclinical models for segmental bone defect research. Biomaterials. 2009; 30 (12): 2149-2163.

27. Pearce AI, Richards RG, Milz S, Schneider E, Pearce SG. Animal models for implant biomaterial research in bone: a review. Eur Cell Mater. 2007; 13: 1-10.

28. Wancket LM. Animal models for evaluation of bone implants and devices: comparative bone structure and common model uses. Vet Pathol. 2015; 52 (5): 842-850.

29. Hamill J, Knutsen K, Denrick T. Biomechanical basis of human movement. 4th edition. Philadelphia: Wolters Kluwer; 2015. 
30. Perry J, Burnfield J. Gait analysis: normal and pathological function. J Sports Sci Med. 2010; 9 (2): 353.

31. Acevedo JI, Sammarco VJ, Boucher HR, Parks BG, Schon LC, Myerson MS. Mechanical comparison of cyclic loading in five different first metatarsal shaft osteotomies. Foot Ankle Int. 2002; 23 (8): 711-716.

32. Fernández-Seguín LM, Diaz Mancha JA, Sánchez Rodríguez R, Escamilla Martínez E, Gómez Martín B, Ramos Ortega J. Comparison of plantar pressures and contact area between normal and cavus foot. Gait Posture. 2014; 39 (2): 789-792.

33. Hills AP, Hennig EM, McDonald M, Bar-Or O. Plantar pressure differences between obese and non-obese adults: a biomechanical analysis. Int J Obes Relat Metab Disord. 2001; 25 (11): 1674-1679.

34. Jacob HA. Forces acting in the forefoot during normal gait--an estimate. Clin Biomech (Bristol, Avon). 2001; 16 (9): 783-792.

35. Stokes IA, Hutton WC, Stott JR. Forces acting on the metatarsals during normal walking. J Anat. 1979; 129 (Pt 3): 579-590.

36. Luger EJ, Nissan M, Karpf A, Steinberg EL, Dekel S. Patterns of weight distribution under the metatarsal heads. J Bone Joint Surg Br. 1999; 81 (2): 199-202.

37. Richter M, Lintz F, Netto CC, Barg A, Burssens A, Ellis S. Weight bearing cone beam computed tomography (WBCT) in the foot and Ankle: a scientific, technical and clinical guide. Switzerland: Springer; 2020.

38. Martínez-Nova A, Pascual Huerta J, Sánchez-Rodríguez R. Cadence, age, and weight as determinants of forefoot plantar pressures using the Biofoot in-shoe system. J Am Podiatr Med Assoc. 2008; 98 (4): 302-310.

39. Geng X, Shi J, Chen W, Ma X, Wang X, Zhang C et al. Impact of first metatarsal shortening on forefoot loading pattern: a finite element model study. BMC Musculoskelet Disord. 2019; 20 (1): 625.

40. Amemiya A, Okonogi R, Yamakawa H, Susumu K, Jitsuishi $\mathrm{T}$, Sugawara $\mathrm{H}$ et al. The external force associated with callus formation under the first metatarsal head is reduced by wearing rocker sole shoes. Annu Int Conf IEEE Eng Med Biol Soc. 2017; 2017: 4487-4490.

41. Bohnert L, Radeideh A, Bigolin G, Gautier E, Lottenbach M. Mechanical testing of maximal shift scarf osteotomy with insideout plating compared to classic scarf osteotomy with double screw fixation. J Foot Ankle Surg. 2018; 57 (6): 1056-1058.

42. Lawless MW, Reveal GT, Laughlin RT. Foot pressures during gait: a comparison of techniques for reducing pressure points. Foot Ankle Int. 2001; 22 (7): 594-597.

43. Mohd Khirul HM, Faizal MF. Composite patch repair using natural fiber for aerospace applications, sustainable composites for aerospace applications. Science Direct. 2018; 171-209. Available in: https://doi.org/10.1016/B978-0-08102131-6.00009-8

44. Crist BD, Borrelli J Jr, Harvey EJ. Essential biomechanics for orthopaedic trauma. A case base guide. 2nd edition. Switzerland: Springer; 2017.

45. Betts DC, Müller R. Mechanical regulation of bone regeneration: theories, models, and experiments. Front Endocrinol (Lausanne). 2014; 5: 211. 